\section{La chimiokine CXCL12 \\ et son récepteur CXCR4 \\ dans le contrôle des infections par les papillomavirus humains}

\section{Nouveaux facteurs de susceptibilité à la pathogénie virale}

Floriane Meuris ${ }^{1}$, Agnieszka Jaracz-Ros ${ }^{1}$, Françoise Gaudin ${ }^{1,2}$, Géraldine Schlecht-Louf ${ }^{1}$, Claire Deback ${ }^{1}$, Françoise Bachelerie ${ }^{1}$
IInflammation, chimiokines et immunopathologie, Inserm UMR 996, Fac. de médecine-Univ Paris-Sud, Université Paris-Saclay, Clamart, France. ${ }^{2}$ US31-UMS3679, Plateforme PHIC, Institut Paris-Saclay d'Innovation Thérapeutique (IPSIT), Inserm, CNRS, Univ Paris-Sud, Université Paris-Saclay, Clamart France. francoise.bachelerie@u-psud.fr
> Les papillomavirus constituent une large famille de petits virus à ADN ayant co-évolué avec leurs hôtes (i.e. mammifères, oiseaux, reptiles et marsupiaux) dont ils infectent sélectivement les épithéliums malpighiens (comme la peau et les muqueuses), le plus souvent de manière asymptomatique [1]. De nombreux papillomavirus humains (HPV), près de 200 génotypes rapportés à ce jour, ont été isolés d'individus sains et identifiés comme des commensaux ${ }^{1}$ appartenant au microbiome viral [2]. L'existence de cet écosystème viral, révélé sur plusieurs sites anatomiques (comme la peau et les muqueuses oropharyngées et anogénitales) par des approches de séquençage métagénomique, est cohérent avec les caractéristiques du cycle biologique des papillomavirus. Leur réplication est en effet non-lytique et faiblement immunogène, une conséquence de leur dépendance aux processus de prolifération, de différenciation et de renouvellement des kératinocytes. Se pose la question de la physiopathologie de cet écosystème, de sa persistance et de son évolution en relation avec le statut immunologique de l'hôte.

Un organisme commensal est un organisme qui vit aux dépens d'un autre, mais qui ne lui cause pas de dommage.
Pathogénie des papillomavirus humains La diversité de la communauté des papillomavirus humains et la coexistence de plusieurs génotypes au sein de lésions suggèrent l'existence de facteurs viraux et cellulaires pouvant influencer la persistance virale (comme l'interférence virale ou l'immunité croisée) [3] et, notamment, celle des génotypes considérés à haut risque (hr) oncogène. Les infections par HPV sont en effet étudiées pour leur rôle bien établi dans le développement de la majorité des cancers du col de l'utérus, de la plupart des autres cancers ano-génitaux et d'une partie des cancers oropharyngés. Les génotypes en cause dans les infections cutanées se manifestent sous la forme de lésions le plus souvent bénignes (comme les verrues). D'autres génotypes sont associés à des carcinomes cutanés de type non-mélanome à la faveur d'immunodépressions et sont considérés comme des facteurs de risque au développement de ces tumeurs dans la population générale. Responsables de près d'un demi-million de morts dans le monde chaque année, les cancers liés aux papillomavirus demeurent un enjeu de santé publique. Les programmes actuels de prévention des cancers fondés sur la vaccination sont un succès, mais ils ne sont accessibles qu'à certaines populations et leur fenêtre de protection est restreinte à certains génotypes HPV.
Physiopathologie des papillomavirus humains: des indices apportés par les immunodéficiences

Le devenir d'une infection virale est déterminé par les interactions qui s'établissent entre les protéines virales et celles de l'hôte. Ces interactions sont influencées à la fois par des facteurs intrinsèques (tels que la cellule cible et sa réponse à l'infection) et extrinsèques (comme le statut immunologique de l'hôte). Pour certains génotypes muqueux, plus particulièrement étudiés en raison de leur association avec les cancers cervicaux (comme les papillomavirus à haut risque de type 16 et 18 , hrHPV16 et 18), l'expression dérégulée et persistante des oncogènes viraux et l'inefficacité de la réponse immunitaire sont des facteurs de risque pour la progression maligne. L'infection, à l'origine productive et asymptomatique, se transforme séquentiellement en une lésion bénigne, puis précancéreuse (dysplasie) ou cancéreuse (néoplasie) (Figure I). Les génotypes HPV infectant l'épithélium cervical ne développeraient leur potentiel oncogène que dans certaines populations cellulaires de la jonction située entre l'exocol ${ }^{2}$ et l'endocol [4],

${ }^{2}$ Le col de l'utérus ou cervix est constitué de l'exocol, partie externe du col visible à l'examen, et l'endocol, partie interne du col de l'utérus permettant de faire communiquer l'exocol et l'endomètre (couche de cellules recouvrant l'intérieur de l'utérus). 


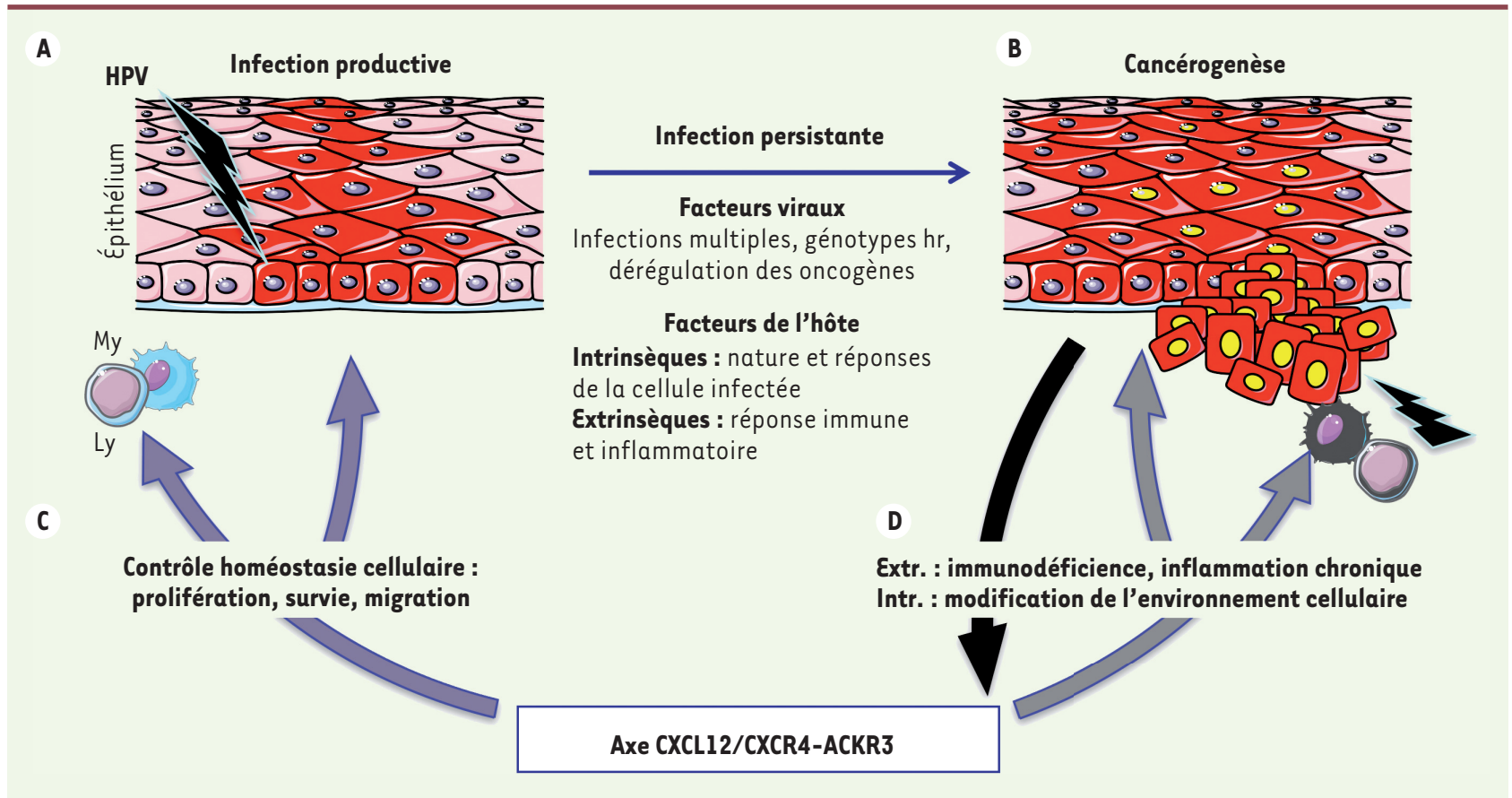

Figure 1. Rôles possibles de l'axe de signalisation formé par la chimiokine CXCL12 et ses récepteurs dans le cycle biologique des papillomavirus humains. Les papillomavirus humains (HPV) ont un tropisme pour les épithéliums malpighiens. A. Le virus infecte les kératinocytes de la couche basale (cellules rouges) et son cycle réplicatif est lié au programme de différenciation cellulaire. Celui-ci s'effectue lors de la migration des kératinocytes vers la surface de l'épiderme jusqu'à la desquamation de la couche cornée. L'infection productive, non-lytique et faiblement immunogène, est le plus souvent asymptomatique. $\boldsymbol{B}$. Des facteurs viraux et de l'hôte maintiennent cet écosystème viral. Une rupture de cet équilibre engendre la persistance virale et l'apparition de lésions bénignes qui sont le plus souvent résolutives. $C$. La persistance de virus à haut risque oncogène (hr) peut évoluer vers la dysplasie et la cancérogenèse. L'axe CXCL12/CXCR4-ACKR3 participe au contrôle du cycle biologique d'HPV en régulant la prolifération des kératinocytes et la migration des cellules immunitaires (lymphocytes, Ly et cellules myéloïdes, My). D. Des dysfonctions de cet axe, révélées dans des immunodéficiences rares, agissent à la fois aux niveaux intrinsèque (Intr.) et extrinsèque ( $E x t r$.$) via la mise en$ place d'une inflammation chronique. Elles conduisent à la dérégulation de l'expression des oncogènes (cellules rouges à noyaux jaunes) et, via une boucle autocrine (flèche noire), à la carcinogenèse (effraction de la lame basale). CXCL12/CXCR4-ACKR3: C-X-C motif chemokine 12/C-X-C chemokine receptor type 4-atypical chemokine receptor 3.

suggérant l'importance de la nature de la cellule infectée et, plus généralement, la contribution de facteurs de susceptibilité à la pathogenèse virale. Comment ces mécanismes peuvent-ils être extrapolés à d'autres génotypes, et notamment à ceux responsables des infections cutanées, reste cependant une question ouverte compte tenu de la complexité d'étude de la biologie des papillomavirus [1]. À cet égard, la susceptibilité sélective à la pathogénie HPV qui caractérise certains syndromes d'immunodéficiences primaires (comme les lésions cutanées ou ano-génitales persistantes et le plus souvent can- céreuses) [5], peut apporter des éléments de compréhension. C'est le cas de l'épidermodysplasie verruciforme, qui se manifeste par l'apparition de nombreuses verrues pouvant dégénérer en des cancers de la peau dans les zones plus particulièrement exposées au soleil. L'association étiologique de ce syndrome avec des mutations des gènes codant les protéines EVER (epidermodysplasia verruciformis) 1 et 2 a démontré les rôles de celles-ci à la fois dans les réponses cellulaires (comme I'homéostasie du zinc) et systémiques de l'hôte (en particulier immunitaires) contre certains génotypes viraux. C'est également le cas moins étudié du WHIM ${ }^{3}$, un syndrome d'immunodéficience très rare, dont un des signes cardinaux est une susceptibilité sélective aux infections par les HPV, se révélant dans plus de $70 \%$ des cas par le développement de verrues cutanées étendues et de papillomes ano-génitaux qui évoluent en cancers [6].

\footnotetext{
${ }^{3}$ Le syndrome WHIM (Warts - hypogammaglobulinemia infections - myelokathexis) est un déficit immunitaire autosomique dominant congénital principalement caractérisé par une lymphopénie, une neutropénie et une myélokathexie (présence anormalement élevée de neutrophiles matures dans la moelle osseuse) associées à une susceptibilité aux infections par les papillomavirus humains.
} 
Dérégulation de la chimiokine CXCL12 et de ses récepteurs comme facteur de susceptibilité à la pathogénie virale Le syndrome WHIM (SW) est causé par des mutations du récepteur CXCR4 (C-X-C chemokine receptor type 4) de la chimiokine $\mathrm{CXCL12}$ ( $C-X-C$ motif chemokine 12), un récepteur couplé aux protéines $G^{4}$. Ces mutations entraînent un gain de fonction du récepteur et une altération du processus de désensibilisation (c'est-à-dire l'inactivation des protéines $G$ et l'internalisation du récepteur). L'axe de signalisation CXCL12/CXCR4 est modulé par CXCR7 (ou ACKR3, atypical chemokine receptor 3 ), le second récepteur atypique de $\mathrm{CXCL12}$, très conservé chez les vertébrés. Cet axe a été caractérisé dans des modèles murins comme étant essentiel au développement embryonnaire et impliqué dans des processus homéostatiques mais aussi inflammatoires, infectieux et cancéreux à l'âge adulte. L'expression de CXCL12 et de ses deux récepteurs n'est en effet pas restreinte aux cellules hématopoïétiques. Au-delà de ses fonctions princeps dans le trafic des leucocytes entre le sang périphérique et les tissus lymphoïdes, ce trio participe à la régulation de l'adhérence, la chimiotaxie, la survie et la prolifération de nombreux types cellulaires [7]. Un modèle murin original du SW a apporté la démonstration du rôle étiologique des dysfonctions de CXCR4 dans la pan-leucopénie caractéristique du syndrome. Ces dysfonctions font suite à des anomalies de développement, de localisation et de migration des cellules lymphoïdes et myéloïdes. La contribution de ces anomalies à la pathogénie virale s'exerce au niveau extrinsèque, traduisant un rôle de ces cellules dans la réponse immune, comme le suggère la rémission d'un patient SW suite à un accident géné-

${ }^{4}$ CXCL12 est une chimiokine de type CXC ( $C-X-C$ motif) qui se lie aux récepteurs CXCR4 et CXCR7, également nommé ACKR3. tique rare (une chromothripsis ${ }^{5}$ ) qui a eu pour bénéfice une reconstitution de la lignée myéloïde [8]. Elle s'exerce aussi localement, en entretenant une inflammation chronique des épithéliums infectés, favorisant ainsi la néoplasie induite par les virus à haut risque oncogène hrHPV [9].

De façon plus inattendue, nous avons mis en évidence un rôle de $\mathrm{CXCL} 12$ et de ses récepteurs dans la pathogénie d'HPV au niveau intrinsèque, c'est à dire à l'échelle du kératinocyte infecté (Figure 1). En effet, la présence anormale de CXCL12 dans les lésions HPV cutanées et muqueuses provenant de patients atteints du SW mais aussi d'individus issus de la population générale, nous a permis de formuler I'hypothèse selon laquelle l'axe CXCL12/CXCR4-ACKR3 pouvait participer à la pathogénie virale. L'activation autocrine de cet axe de signalisation, qui s'instaure dans des kératinocytes primaires immortalisés par hrHPV 8 ou 16 comme conséquence de l'expression des oncogènes viraux, s'avère être essentielle à la prolifération, la survie et la migration de ces cellules. Quand ce processus prend place dans le contexte de l'expression du mutant gain de fonction de CXCR4 associé au SW (c'est-à-dire CXCR $4^{\text {WHIM }}$ ), il confère à ces kératinocytes immortalisés des propriétés transformantes in vivo qu'ils n’ont pas lorsqu'ils expriment le récepteur CXCR4 sauvage

\section{$[10,11](\rightarrow)$.}

Ces résultats iden-

tifient donc les dys-

\section{$(\rightarrow)$ Voir la Nouvelle} de $\varepsilon$. Brotin et al., $m / s n^{\circ} 4$, avril 2011, page 341
Rôle de l'axe CXCL12/CXCR4 dans le contrôle de la réplication virale

Afin de déterminer le rôle de l'axe CXCL12/CXCR4 dans le contrôle du cycle réplicatif des papillomavirus, nous avons développé des cultures organotypiques tridimensionnelles (3D) de kératinocytes. Ces cultures ont constitué un jalon important de l'étude de la biologie de ces virus dont la réplication est étroitement liée à la dynamique de prolifération/différenciation des kératinocytes [12]. Les cultures 3D de kératinocytes exprimant le récepteur CXCR4 sauvage permettent le cycle réplicatif complet d'hrHPV18, associé à un profil d'expression finement régulé des protéines virales jusqu'à la production de virions infectieux. En revanche, bien que I'expression du mutant CXCR $4^{\text {WHIM }}$ ne modifie pas la topologie subcellulaire des cultures 3D, elle dérégule l'expression des oncogènes viraux, favorisant leur stabilisation aux dépens de la réplication virale. Ces dérégulations sont associées à des changements cellulaires comme l'altération de la prolifération et de la mort cellulaire indicatifs d'un processus de carcinogenèse en cours. Bloquer le gain de fonction du récepteur mutant, notamment en utilisant un antagoniste sélectif de CXCR4 (comme I'AMD3100) lors de la stratification des cultures 3D, inverse ce processus, normalisant la prolifération cellulaire et le patron d'expression des protéines virales associées au cycle réplicatif d'HPV [12].

\section{Conclusions}

L'ensemble de ces données révèle que CXCL12 et ses récepteurs participent au contrôle exercé par l'hôte sur le cycle biologique des papillomavirus comme conséquence de leurs fonctions dans I’homéostasie épithéliale (telles la prolifération et la survie des kératinocytes) et immunologiques (comme la migration et la localisation des leucocytes) et apparaissent ainsi comme de nouveaux facteurs de susceptibilité à la pathogé- comme des cofacteurs de la carcinogénèse associée aux papillomavirus. Ils sion et de fonction de CXCL12 et de récepteurs, rapportées dans différents types de tumeurs, et qui participent au développement et à la dissémination de celles-ci [7].

${ }^{5}$ chromothripsis : remaniement chromosomique massif. font écho aux dérégulations d'expre

\section{nie virale. $\diamond$}


The CXCL12/CXCR4 signaling pathway

in the control of human papillomavirus

infection: new susceptibility factors in

viral pathogenesis

\section{LIENS D'INTÉRÊT}

Les auteurs déclarent n'avoir aucun lien d'intérêt concernant les données publiées dans cet article.

\section{RéFÉRENCES}

1. Doorbar J, Egawa N, Griffin H, et al. Human papillomavirus molecular biology and disease association. Rev Med Virol $2015 ; 25: 2-23$.

2. Foulongne V, Sauvage V, Hebert C, et al. Human skin microbiota: high diversity of DNA viruses identified on the human skin by high throughput sequencing. PLoS One 2012 ; 7 : e38499.
3. Bzhalava D, Muhr LS, Lagheden C, et al. Deep sequencing extends the diversity of human papillomaviruses in human skin. Sci Rep $2014 ; 4$ : 5807.

4. Herfs M, Yamamoto Y, Laury A, et al. A discrete population of squamocolumnar junction cells implicated in the pathogenesis of cervical cancer. Proc Natl Acad Sci USA 2012 ; 109 : 10516-21.

5. Leiding JW, Holland SM. Warts, et al. Human papillomavirus in primary immunodeficiencies. J Allergy Clin Immun 2012; 130 : 1030-48.

6. Beaussant Cohen $S$, Fenneteau 0 , Plouvier $\varepsilon$, et al. Description and outcome of a cohort of 8 patients with WHIM syndrome from the French severe chronic neutropenia registry. Orphanet J Rare Dis 2012 ; 7 : 71.

7. Bachelerie F, Ben-Baruch A, Burkhardt AM, et al. International union of basic and clinical pharmacology [corrected]. LXXXIX. Update on the extended family of chemokine receptors and introducing a new nomenclature for atypical
NOUVELLE

\section{La dysplasie épithéliale intestinale, ou quand l'intestin est sous 《deux de tension 》 cellulaire}

Cécile Gaston $^{1}$, Julie Salomon ${ }^{1,2}$, Olivier Goulet ${ }^{2}$, Delphine Delacour ${ }^{1}$
L'épithélium intestinal, une monocouche épithéliale polarisée dynamique

Une monocouche épithéliale est sujette au remodelage lors du développement ou d'événements de réparation tissulaire; un renouvellement cellulaire, des réarrangements ainsi que des phénomènes d'extrusion adviennent constamment pour maintenir l'homéostasie de cette monocouche. Un épithélium doit donc stabiliser son organisation et s'adapter de façon globale, pour maintenir son état polarisé et cohésif.

L'épithélium intestinal constitue un très bon modèle pour étudier différentes questions d'organisation tissulaire et cellulaire. La monocouche de cellules épithéliales qui tapisse l'intestin grêle présente une architecture simple et régulière, où les cellules prolifératives et les cellules différenciées sont distribuées dans des compartiments tissulaires différents: respectivement, les «cryptes » et les «villosités ». Tandis que les entérocytes évoluent le long de la villosité, les cellules suivent un processus d'organisation spécifique, appelé différenciation terminale, avec la formation d'une structure fonctionnelle au cortex apical, la bordure en brosse des microvillosités; celle-ci expose les enzymes intestinales, augmente la surface membranaire au pôle apical et potentialise ainsi l'absorption de nutriments à l'interface entre la lumière de l'intestin et l'épithélium [1, 2].

\section{EpCAM, un acteur clé dans le maintien} de la monocouche épithéliale intestinale Les entéropathies congénitales présentent des perturbations de l'organi- chemokine receptors. Pharmacol Rev 2014 ; 66 : 1-79.

8. McDermott DH, Gao JL, Liu $Q$, et al. Chromothriptic cure of WHIM syndrome. Cell $2015 ; 160: 686-99$.

9. Meuris F, Gaudin F, Aknin ML, et al. Symptomatic improvement in human papillomavirus-induced epithelial neoplasia by specific targeting of the CXCR4 chemokine receptor. J Invest Dermatol 2016 ; $136: 473-80$.

10. Chow Ky, Brotin $\varepsilon$, Ben Khalifa $Y$, et al. A pivotal role for CXCL12 signaling in HPV-mediated transformation of keratinocytes: clues to understanding HPV-pathogenesis in WHIM syndrome. Cell Host Microbe $2010 ; 8: 523-33$.

11. Brotin $\varepsilon$, Carthagena L, Chow KYC et al. La chimiokine CXCL12 et le papillomavirus humain. Med Sci (Paris) $2011 ; 27: 341-3$.

12. Meuris F, Carthagena L, Jaracz-Ros A, et al. The CXCL12/CXCR4 signaling pathway: a new susceptibility factor in human papillomavirus pathogenesis. PLoS Pathog 2016 ; 12 : e1006039.

\author{
${ }^{1}$ Adhérence cellulaire et mécanique, Institut \\ Jacques Monod, CNRS UMR7592, Université \\ Paris Diderot, 15, rue Hélène Brion, 75013 \\ Paris, France. \\ ${ }^{2}$ Département de gastroentérologie \\ pédiatrique et nutrition, Hôpital Necker- \\ Enfants Malades, Sorbonne Paris Cité, \\ 149, rue de Sèvres, 75015 Paris, France. \\ delphine.delacour@ijm.fr
}

sation de l'épithélium intestinal le long de l'axe crypto-villositaire et sont, pour la plupart, responsables d'insuffisance intestinale. Deux d'entre elles sont particulièrement sévères : I'atrophie microvillositaire (AMV) et la dysplasie épithéliale intestinale (DEl en français, CTE pour congenital tufting enteropathy, en anglais) [3].

La DEI a été décrite pour la première fois en 1994 [4] et plus en détail en 1995 [5]. Cette entéropathie est moins fréquente que I'AMV, avec une incidence estimée à $1 / 200000$ en Europe. La DEl est histologiquement définie par un épithélium dysplasique caractéristique, prenant de façon hétérogène un aspect pseudostratifié où les entérocytes s'accumulent sous forme de houppettes ou «tufts» [6], qui touchent jusqu'à $70 \%$ des villosités à un stade avancé [6]. 